\title{
Sistema de tareas didácticas interactivas para la enseñanza del inglés con propósitos específicos médicos en medicina natural y tradicional
}

\author{
Daimy Delgado Ávila*
}

\begin{abstract}
RESUMEN
El objetivo de la presente investigación es observar el sistema de tareas didácticas interactivas para la asignatura inglés IX que corresponde al ciclo de inglés con propósitos específicos médicos basados en el uso de la medicina natural y tradicional. Se considera una muestra de estudiantes de quinto año de la Carrera de Medicina, de la Filial de Ciencias Médicas Comandante Manuel Piti Fajardo, del Municipio San Cristóbal, Artemisa, Cuba. Como resultado se obtuvo un material complementario para el desarrollo de las clases de inglés con propósitos específicos médicos, brindando especial atención al vocabulario y a las funciones comunicativas a utilizarse en la entrevista doctor-paciente, doctor-doctor, reportes de casos y presentación de seminarios.
\end{abstract}

Palabras clave: sistema de tareas, didáctica interactiva, inglés con propósitos específicos médicos.

System of interactive tasks for teaching english for medical specific purposes in natural and traditional Medicine

\begin{abstract}
The objective of this research is to observe the system of interactive teaching tasks for English subject in the cycle corresponding to English for specific medical purposes based on the use of natural and traditional medicine. It considers a sample of students in the fifth year of the School of Medicine of the Medical Sciences School Commander Manuel Piti Fajardo, from the municipality of San Cristobal, Artemisa, Cuba. As a result, it obtained a supplementary material for the development of English classes for specific medical purposes, with special attention to vocabulary and communicative use in the doctor-patient interview and doctor - doctor relations, case reports and presentation of seminars.
\end{abstract}

Key words: System of tasks, interactive didactics, English for specific medical purposes.

* Daimy Delgado Ávila es Máster em Educación Superior, Licenciada en Lengua Inglesa, Profesora Auxiliar Filial de Ciencias Médicas Comandante Manuel Piti Fajardo, Departamento de Idiomas, MINSAP, San Cristóbal, Artemisa, Cuba. E-mail: daimy10@infomed.sld.cu 


\section{Introducción}

Los avances de la ciencia y la tecnología en el mundo actual exigen a los profesionales una preparación constante y de calidad. Inmersos en el proceso de enseñanza aprendizaje los profesionales de la Pedagogía trabajan cada día por alcanzar mejores estándares de calidad en este proceso.

En las carreras de las Ciencias Médicas en Cuba, dígase Medicina, Estomatología, Psicología Médica, Enfermería y Tecnología de la Salud se imparte la disciplina de inglés; la misma se estructura de forma diferente en cada carrera según su diseño curricular. En la Carrera de Medicina, los estudiantes reciben inglés general en primero, segundo y tercer año, siendo los cursos distribuidos en 6 semestres (Inglés I, II, III, IV, V y VI); los que corresponden al ciclo de inglés general. Se utiliza como bibliografía básica la serie pedagógica diseñada por un colectivo de autores cubanos Vision one, Two y three. En cuarto y quinto año, los estudiantes reciben inglés VII, VIII, IX y X, pertenecientes al ciclo de inglés con propósitos específicos médicos, distribuidos en 4 semestres. En este caso, la bibliografía básica a emplear corresponde, también, a una serie pedagógica: English through medicine one, para cuarto año, y English through medicine two, para quinto año.

En el proceso de enseñanza y aprendizaje en las universidades de Ciencias Médicas cubanas se reconoce la importancia política, económica, social y cultural de las lenguas extranjeras, especialmente el inglés. Por ello, su enseñanza es vital dentro de la estrategia y cumplimiento de los propósitos internacionalistas de la sociedad cubana. La asignatura contribuye a la formación científica del mundo en los alumnos, al tomar como punto de partida el lenguaje como fenómeno social y la relación pensamiento, lenguaje y cultura, así como al proporcionar el análisis de la lengua para penetrar en su esencia.

El objetivo integrador de la asignatura inglés IX es comunicarse profesionalmente en idioma inglés en situaciones reales y simuladas, utilizando las cuatro habilidades de la lengua inglesa; las funciones comunicativas específicas en cada actividad de la práctica médica; el vocabulario técnico, desarrollando el colectivismo, el espíritu de 
consagración al estudio y al trabajo, la motivación por la profesión, la participación en eventos científicos, el respeto por los principios éticos y morales de la sociedad socialista y el sentimiento humanista en aras de fomentar la paz, la solidaridad, la coexistencia pacífica y la colaboración con otros pueblos.

\section{Definición de inglés con propósitos específicos}

Es imposible para dar una definición de inglés con propósitos específicos (ESP) dentro de la enseñanza de lenguas extranjeras, que se pueda aplicar de manera universal, aunque se coincide en la importancia del análisis de las necesidades como punto de partida para alcanzar la meta que se propone en un período de tiempo determinado.

A continuación, se exploran algunas de las definiciones de ESP aportadas a nivel mundial. Según Hutchinson y Waters (1987), en el ESP las decisiones de contenido y método se toman en relación a las razones del aprendiz por aprender. Por su lado, Gatehouse (200I) presenta la definición aportada por Strevens (I988). Este autor define ESP al identificar sus características absolutas y variables. La definición de Strevens hace una distinción entre cuatro características absolutas y dos variables: las características absolutas son que ESP consiste en la enseñanza de idioma inglés, la cual está diseñada para satisfacer las necesidades más específicas del estudiante, relacionadas al contenido con disciplinas particulares, ocupaciones y actividades, y centradas en el idioma apropiado para dichas actividades en cuanto a sintaxis, léxico, discurso, semántica, y análisis de este. Las características variables de ESP indican que puede estar restringido a las habilidades idiomáticas a aprender y no enseñarse de acuerdo con ninguna metodología preestablecida. Sin embargo, en los últimos años se evidencian tendencias dentro del ESP, específicamente, el método Aprendizaje Integrado de Conocimientos curriculares y Lengua Extranjera, AICLE (Fernández, 200I), que se integra armónicamente con el enfoque comunicativo.

Anthony (1997), por otra parte, señala que ha habido considerable debate en torno al significado de ESP, a pesar del hecho de ser 
un enfoque bastante utilizado durante las últimas tres décadas. Dudley-Evans y Saint John (1998), en tanto, ofrecen una definición modificada. ESP se define para satisfacer necesidades específicas del estudiante; hace uso de la metodología y las actividades de la disciplina a la que sirve; $y$ se centra en el idioma, habilidades, discurso y género apropiados para estas actividades. Continúan planteando que ESP puede estar relacionado o diseñado para disciplinas específicas; puede utilizarse, en situaciones instructivas específicas, una metodología diferente a la del inglés general. En general, está diseñado para adultos, ya sea en el nivel superior o en una situación profesional; no obstante, pudiera, también, estar dirigido al nivel medio y principiante.

\section{Metodología}

Los criterios antes expuestos sirvieron a la autora como punto de partida para la elaboración del sistema de tareas didácticas interactivas en inglés con propósitos específicos médicos. Al analizar el programa de la asignatura inglés IX, se constató que la misma está estructurada en cinco unidades didácticas. Unidad I Benign Prostatic Hyperplasia (Hiperplasia Benigna de Próstata); Unidad 2 Jaundice (Ictericia); Unidad 3 Tropical Diseases (Enfermedades Tropicales), Unidad 4 Alcobolism (Alcoholismo) y Unidad 5 Childhood Infectious Diseases (Enfermedades Infecciosas en la Infancia).

La bibliografía básica que se utiliza para impartir esta asignatura es English tbrough medicine two), la cual está muy bien diseñada y da cuenta de los contenidos fundamentales de cada Unidad. Además, hace énfasis en el tratamiento medicamentoso, no así con el uso de la medicina natural y tradicional, siendo esta de relevancia en los momentos actuales que vive la sociedad cubana y latinoamericana.

Teniendo en cuenta los objetivos y contenidos del programa, se realizó una búsqueda bibliográfica y una actualización en los tratamientos de medicina natural y tradicional para las patologías que se presentan en el programa. También, se efectuaron entrevistas y encuestas a personas mayores de la localidad, quienes tienen un conocimiento popular sobre el uso de la medicina natural y tradicional. Después de consultar los 
resultados obtenidos con médicos altamente calificados y con maestrías en el tema en cuestión, se prosiguió a la elaboración del sistema de tareas didácticas interactivas para la enseñanza del inglés con propósitos específicos médicos en la asignatura de inglés IX.

La aplicación se efectuó con una muestra de estudiantes de quinto año de la Carrera de Medicina, de la Filial de Ciencias Médicas Comandante Manuel Piti Fajardo, del Municipio San Cristóbal, Artemisa, Cuba.

\section{La didáctica interactiva de lenguas}

En la didáctica interactiva el maestro y los alumnos asumen nuevas y complejas funciones. La clase interactiva de excelencia se define como un proceso formativo, interactivo, comunicativo, socio-cultural, holístico e integrador, con una fuerte base cognitiva, y esencialmente humanista, en el cual un grupo de alumnos disfruta y se responsabiliza en condiciones favorables de aprendizaje con la solución de tareas que satisfacen sus necesidades, en un contexto de socialización y cooperación, que les permite autorrealizarse y experimentar cambios duraderos en su actitud, actuación y pensamiento, transferibles a nuevas situaciones, creados por la actividad práctica reflexiva en un proceso de desarrollo de competencias y formación de valores, creado, facilitado y dirigido por el maestro (Acosta, 2010).

El desafío que se plantea es: ¿cómo diseñar un sistema de tareas didácticas interactivas para la enseñanza del inglés con propósitos específicos médicos, a través de la medicina natural y tradicional, para estudiantes de quinto año de la carrera de medicina? Existen diferentes tipos de actividades recomendables para la didáctica interactiva de lenguas en la Educación Superior, tales como: trabajos de proyectos, tareas interactivas en pequeños grupos, trabajo en parejas, aprendizaje en diversos escenarios, uso de páginas web, software, e-mails, interacción de los estudiantes con usuarios, simulaciones, juegos de roles, el arte: dramas, música, cine, pinturas; las presentaciones en público de discursos.

Ahora bien, otro desafío complejo de resolver es: ¿cuál de estos utilizar teniendo en cuenta las características específicas de los estudiantes de quinto año de medicina, sus intereses, necesidades y 
motivaciones personales y colectivas? El factor psicológico constituye un eslabón fundamental cuando se trata del aprendizaje de una lengua extranjera, así como la motivación que los estudiantes sienten hacia el aprendizaje del inglés con propósitos específicos médicos es de vital importancia en el proceso de enseñanza aprendizaje.

\section{Sistema de tareas didácticas interactivas para la enseñanza del inglés con propósitos específicos médicos a través de la medicina natural y tradicional para estudiantes de quinto año de la Carrera de Medicina}

A continuación, se presenta una tabla con cada Unidad del Programa de la asignatura y las enfermedades que se estudian en cada una de ellas. Es necesario especificar que en algunas unidades solo se estudian las enfermedades que aparecen como tema principal de la Unidad.

Tabla I. Unidades del Programa y enfermedades estudiadas

\begin{tabular}{|l|l|}
\hline \multicolumn{1}{|c|}{ UNIDADES } & \multicolumn{1}{c|}{ ENFERMEDADES } \\
\hline $\begin{array}{l}\text { Unidad I. Benign Prostatic Hyperplasia } \\
\text { (Hiperplasia Benigna de Próstata). }\end{array}$ & \\
\hline Unidad 2. Jaundice (Ictericia). & $\begin{array}{l}\text { Cirrhosis of the Liver (Cirrosis Hepática), } \\
\text { Pancreatitis y Hepatitis. }\end{array}$ \\
\hline $\begin{array}{l}\text { Unidad 3. Tropical Diseases } \\
\text { (Enfermedades Tropicales). }\end{array}$ & $\begin{array}{l}\text { Malaria, Dengue, Cólera y } \\
\text { Leptospirosis. }\end{array}$ \\
\hline Unidad 4. Alcobolism (Alcoholismo). & Paperas, Sarampión y Varicela. \\
\hline $\begin{array}{l}\text { Unidad 5. Childhood Infectious Diseases } \\
\text { (Enfermedades Infecciosas en la } \\
\text { Infancia). }\end{array}$ & . \\
\hline
\end{tabular}

El sistema de tareas fue diseñado sobre la base de las unidades didácticas planteadas en el Programa y con una secuencia lógica y progresiva, una en relación con la otra. En total se diseñaron doce tareas didácticas interactivas. Para ejemplificar este trabajo la autora seleccionó la Unidad 2. Jaundice (Ictericia) y la enfermedad Hepatitis. Cada una de estas tareas se realizan en el marco de la clase y partiendo de los contenidos que corresponden, según Programa y Plan de clase. Todas las actividades planificadas se desarrollan de lo simple a lo complejo. 
Task 3.

a. Look at the letters written in the blackboard. They are jumbled. Work in pairs and try to form a word that has relation with these symptoms and signs.

Symptoms and signs: weakness, yellowish skin, asthenia, pale stools.

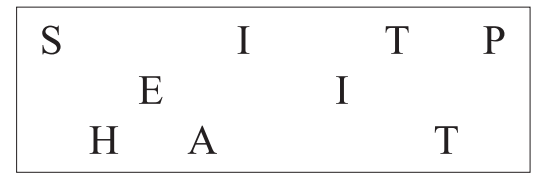

Task 3. b. What is Hepatitis?

Read the following information and organize it. Try to give the correct concept for Hepatitis.

Se forman grupos de 4 estudiantes, pueden ser, por ejemplo, con cuatro palabras que tengan relación con esta enfermedad. Las palabras son hígado, inflamación, amarillo, debilidad, etc. El profesor divide el concepto de hepatitis en oraciones y lo entrega en tarjetas a los alumnos de un mismo equipo. Esto es un ejemplo de jumbled sentences, donde los alumnos trabajan interactuando y utilizando el idioma inglés hasta formar el concepto correctamente. El profesor estimula dando feedback positivo a los estudiantes, pues son el centro de la actividad. El profesor solo monitorea, brinda información y responde las dudas si fuera necesario, además, atiende las diferencias individuales.

Task 3. c. My case report.

Read the following case report and complete it with the information that your classmates bave. Find out the missing information from each other.

Ana Diaz is 32 years old. She is a primary teacher. She came in to the bospital complaining of weakness, the patient also reported pale stools and dark urine. She denies fever. She bad a bealthy life prior to this admission. Her mother is bypertensive and her father died three years ago because of cirrbosis of the liver. She is married and she has one son. She smoked five cigarettes a day. She was taken no medication. On the physical examination.

Esta actividad se realiza en grupos. Se reparten 3 tarjetas con esta información del reporte de caso y el resto de la clase tiene tarjetas 
con pedazos de información, por ejemplo: yellowish skin was found, a urinalysis was performed, an abdominal ultrasaound was indicated, the result was positive, hepatomegaly was found. Los estudiantes tienen que ponerse de pie, caminar, interactuar, hacer preguntas hasta ir encontrando la información necesaria entre sus compañeros para completar el reporte de caso y hacerlo de forma organizada (siguiendo el orden lógico para el reporte de caso). El profesor desde una esquina del aula monitorea la actividad. Esto es un ejemplo de information-gap activity.

\section{Task 3.d.}

The use of natural and traditional medicine is an important feature nowadays in our country. For young doctors, it is a challenge to learn a little bit everyday about this and its management. Walk around the classroom and find your couple. These advices deal with natural and traditional medicine for treating bepatitis. Remember that when you are going to give an advice to the patient you must use the communicative function of giving advice, eg You should.

Card 1. boil.

Card 2. wash the bed clothes

Card 3 rest

Card 4 boil the tamarind's leaves

Card 5 avoid

Card 6 eat

Card 7 eat

Card 8 eat

Card 9 eat
Couple Card. the water for drinking.

Couple Card. Everyday.

Couple Card. in bed.

Couple Card. and drink it daily.

Couple Card. eating fats and salt in foods.

Couple Card. and drink it daily.

Couple Card. a lot of papaya's sweets.

Couple Card. lamb chop.

Couple Card. roast fish.

Para el desarrollo de esta actividad se reparten las tarjetas entre los miembros del grupo y estos interactúan utilizando el idioma inglés con propósitos específicos médicos hasta encontrar su pareja. Una vez encontrada la pareja, dicen el consejo que les corresponde al resto del grupo, en voz alta, y el profesor pregunta si conocen de otros usos de la medicina natural y tradicional para curar o aliviar esta enfermedad. 


\section{Conclusiones}

La elaboración y utilización del sistema de tareas didácticas interactivas para la enseñanza del inglés con propósitos específicos médicos, a través de la medicina natural y tradicional, para estudiantes de quinto año de la carrera de medicina, favoreció el desarrollo de la clase práctica de la lengua inglesa con propósitos específicos médicos, pues los estudiantes se sintieron motivados durante todo el proceso docente educativo y adquirieron los conocimientos interactuando entre ellos y aprendieron unos de otros. Además, los estudiantes lograron mejorar su competencia comunicativa y su dominio del inglés con propósitos específicos médicos, lo cual es muy necesario para su futuro desarrollo como profesionales de las ciencias médicas en Cuba. Por último, se observó que los alumnos profundizaron y se actualizaron en los conocimientos que poseían sobre el uso de la medicina natural y tradicional.

\section{Referencias bibliográficas}

Acosta, R. (2010). Didáctica Interactiva de Lenguas. Brasil: Gráfica Lastro.

Anthony, L. (1997). ESP: What does it mean? Recuperado de http://interserver. miyazaki-med.ac.jp/ cue/pc/anthony.htm

Dudley-Evans, T. \& St John, M. (1998). Developments in ESP: A multi-disciplinary approach. Cambridge: Cambridge University Press.

Fernández, A. (200I). Una selección bibliográfica sobre el método AICLE (Aprendizaje Integrado de Conocimientos Curriculares y Lengua Extranjera). Contextos Educativos, 4, 217-239.

Gatehouse, K. (200I). Key Issues in English for specific purposes (ESP). Curriculum Development". TESL Journal, 7 (I0). Recuperado de http:/ / www.cybertrails.com/ jhoey/

Hutchinson, T. \& Waters, A. (1987). English for specific purposes. A learning-centred approach. Cambridge: United Kingdom at the University Press.

Strevens, P. (1988). Languages for Specific Purposes. Program design and evaluation. London: Newbury House. 